\title{
Spontaneous micro-modification of single- layer graphene induced by femtosecond laser irradiation
}

Cite as: Appl. Phys. Lett. 111, 241901 (2017); https://doi.org/10.1063/1.4997343

Submitted: 24 July 2017 . Accepted: 22 November 2017 . Published Online: 11 December 2017

Chen Cheng, Ruiyun He, Carolina Romero, Javier R. Vázquez de Aldana, and Feng Chen
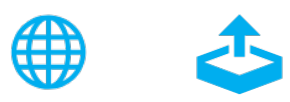

\section{ARTICLES YOU MAY BE INTERESTED IN}

Nanoscale patterning of graphene through femtosecond laser ablation

Applied Physics Letters 104, 053118 (2014); https://doi.org/10.1063/1.4864616

Response of graphene to femtosecond high-intensity laser irradiation

Applied Physics Letters 99, 051912 (2011); https://doi.org/10.1063/1.3623760

Graphene folds by femtosecond laser ablation

Applied Physics Letters 100, 233124 (2012); https://doi.org/10.1063/1.4724213

\section{Challenge us.}

What are your needs for periodic signal detection?

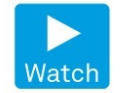

- Zurich

- Instruments

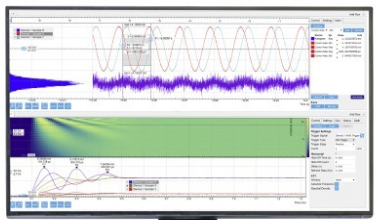

两 


\title{
Spontaneous micro-modification of single-layer graphene induced by femtosecond laser irradiation
}

\author{
Chen Cheng, ${ }^{1}$ Ruiyun He, ${ }^{1}$ Carolina Romero, ${ }^{2}$ Javier R. Vázquez de Aldana, ${ }^{2}$ \\ and Feng Chen ${ }^{1, a)}$ \\ ${ }^{1}$ School of Physics, State Key Laboratory of Crystal Materials, Shandong University, Jinan 250100, China \\ ${ }^{2}$ Laser Microprocessing Group, Universidad de Salamanca, Salamanca 37008, Spain
}

(Received 24 July 2017; accepted 22 November 2017; published online 11 December 2017)

\begin{abstract}
Permanent micro-modifications in single-layer graphene with a spontaneous periodic structural change have been induced by femtosecond (fs) laser irradiation. These modifications present a regular variation along the radial direction from a central ablated region. Based on the obtained micro-Raman spectrum and the reflective micro-spectrum of laser-irradiated graphene, structural modification with periodic variations containing several spectral regimes has been observed, which was in good agreement with periodic topography of the structure observed using an atomic force microscope. It has also been found that several regions of the laser induced structures were with different optical properties, which were identified to be correlated with different modification mechanisms. In addition, after fs laser processing, graphene still maintains crystallinity. This work may be helpful for the development of graphene-based microstructures or devices by fs laser pulses. Published by AIP Publishing. https://doi.org/10.1063/1.4997343
\end{abstract}

Two-dimensional (2D) materials of single-atom-layer scale have drawn great attention for fabrication of various electronic and photonic devices. ${ }^{1}$ Graphene is still one of the most favorable members in the 2D material family due to its excellent properties that are promising for versatile applications. ${ }^{2-4}$ Nevertheless, it is also necessary to modify the specific properties of graphene to satisfy diverse requirements for practical applications. Towards this purpose, modifications of graphene by femtosecond (fs) laser irradiation, which takes advantage of distinct interaction mechanisms with 2D materials to enable extremely localized energy deposition, receive increasing interest of researchers. ${ }^{5,6}$ The irradiation of ultrafast pulsed lasers has been applied in ablative patterning, thinning, and direct writing to $2 \mathrm{D}$ materials. $^{7-9}$ In addition, direct irradiation with fs laser pulses has become a versatile technique to fabricate photonic and optoelectronic devices in bulk transparent materials. ${ }^{10,11}$ The focused fs laser pulses produce localized modifications of micro- or sub-micrometric scales on the surface or inside the materials, in which permanent changes may be created, due to avalanche ionization induced by fs pulses. ${ }^{12-14}$ Recently, the irradiation with fs laser pulses has become an efficient technique for the fabrication and modification of carbonbased materials. ${ }^{15-19}$ For modification of graphene, cutting, doping, defect introduction, folding, etc., have been implemented successfully. ${ }^{14,18-24}$ In addition, the advantage of fs-laser microprocessing of $2 \mathrm{D}$ materials enables costeffective fabrication of micro- or nano-structures towards various devices. ${ }^{20,25,26}$

Nevertheless, the details of localized modification due to the fs laser irradiation on graphene require further investigation in order to obtain a better understanding of the laser-induced effect, which is helpful to realize more precise engineering of the micro- or nanoscale patterns in single-layer materials. In

a)drfchen@sdu.edu.cn this work, we employ fs-laser irradiated graphene on an insulator system and study the spontaneous micro-modification of a single-layer graphene (SLG) irradiated by fs laser pulses. The surface morphology and the Raman spectroscopy have been investigated in order to characterize the fs-laser induced effect on graphene.

Experimentally, in this work, the fs laser beam was focused at the graphene, inducing the ablation at the center and the formation of a series of radial ring structures from the ablated region center. Single-layer graphene was a customized product coated on a $10 \times 10 \mathrm{~mm}^{2}$ surface of an optically polished sapphire wafer, which was produced to be a continuous membrane by chemical vapor deposition (CVD). Graphene was irradiated with a fs pulse laser (amplified Ti:Sapphire laser, 795-nm center wavelength), with a pulse width of $100 \mathrm{fs}$, a repetition rate of $1 \mathrm{kHz}$, and a nearly Gaussian profile $\left(\mathrm{M}^{2}\right.$ at the laser output was $<1.4$ in both axes). The laser beam was focused via a $10 \times$ objective lens (N.A. $=0.3$ ) with a $20 \mathrm{~mm}$-focal-length on the surface of the graphene sample. The irradiation fluence was controlled by using a set of neutral filters and a laser attenuator (consisting of a half wave plate and a linear polarizer) positioned just before the microscope objective. The calculated spot radius was $\sim 2.0 \mu \mathrm{m}$ (assuming a perfect Gaussian beam profile). The graphene sample was mounted on a XYZ motor-driven positioning stage in order to produce a micrometric translation on the three axes. The processing was performed in air at room temperature. The number of pulses incident on each spot was fixed to 1000 , which was controlled with a mechanical shutter. To generate a clear structural boundary, we chose a corresponding peak fluence of $\sim 1 \mathrm{~J} / \mathrm{cm}^{2}$, which was slightly above the multipulse fs ablation thresholds of SLG $\left(0.15 \mathrm{~J} / \mathrm{cm}^{2}\right.$ with 50 -fs-duration) and substrate sapphire $\left(0.89 \mathrm{~J} / \mathrm{cm}^{2}\right.$ with 150 -fs-duration). ${ }^{21,27}$ In this case, the intensity at the central part of the spot was large enough to produce a plasma density exceeding the critical value, leading 
to laser ablation by the well-known Coulomb explosion, which results in a shallow crater in the focal spot center, with the minimal thermal effect to the surrounding membrane. ${ }^{17}$ Via this mechanism, the area of the ablated material can be controlled, and an obvious edge between ablation and surrounding regions can be detected.

The calculated beam fluence at focus is demonstrated in Fig. 1(a). Figure 1(b) shows the surface topography of the crater and the surrounding area observed using an atomic force microscope (AFM). The undulations have a larger extension than the focal spot and present a spatial periodicity along the radial direction from the ablation crater. A scanning electronic microscopy (SEM) image was depicted to show the details [Fig. 1(c)]. Obviously, the fs multi-pulses impacted the sapphire substrate and made a deep pit with a 1.3- $\mu \mathrm{m}$-diameter crater, which was identical to the substrate ablating threshold. A ring area, with a diameter of $\sim 3 \mu \mathrm{m}$, was covered with massive fragments, which was corresponding to the graphene ablating threshold of $0.2 \mathrm{~J} / \mathrm{cm}^{2}$. At this point, it is important to point out that the sample height variations cannot be easily observed using an optical microscope [see Fig. 1(d)]. In contrast, a slightly "polished" area can be found around the irradiated spot, which is marked by a dashed circle in Fig. 1(c). Such a phenomenon, the creation of spontaneous periodic surface structures during laser irradiation, has already been observed in several transparent bulk materials. ${ }^{12,28,29} \mathrm{We}$ consider that a mass of graphene fragments induced by the Coulomb explosion was transferred to other regions by the shock-wave after the explosion. ${ }^{13}$

We employed an optical microscope (Olympus BX43) with integration of a micro-Raman spectrometer to further investigate these spatial periodic modifications. Raman spectroscopy has become a powerful technique to study the microstructure of 2D material research. ${ }^{30}$ It is particularly successful for graphene due to its non-bandgap, which makes incident radiation resonant at all wavelengths, and therefore, the Raman spectrum contains information about both atomic structures and electronic properties. ${ }^{31,32}$ The SLG sample was placed on a 3 -axis motorized stage (0.1- $\mu$ m-resolution) of the microscope system. A $532 \mathrm{~nm}$-laser-beam was focused on the sample by a microscope objective $(100 \times, 0.8 \mathrm{NA})$, (a)

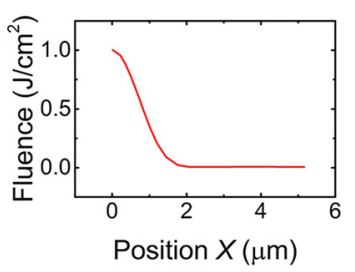

(b)

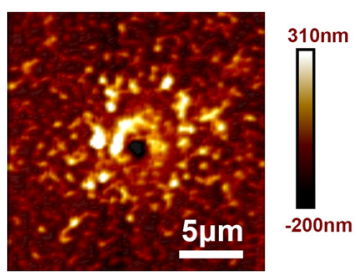

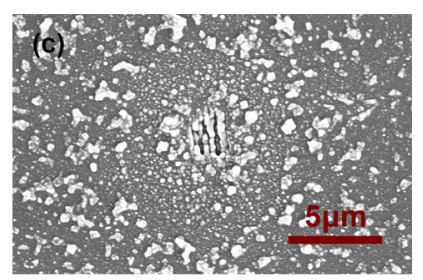

(d)

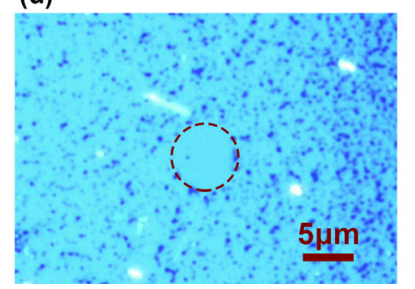

FIG. 1. (a) The calculated fluence at focus. (b) and (c) The AFM image and SEM image of the irradiated region of graphene. (d) The optical microscopy image of the irradiated region. which was used to collect scattered light. After the objective, a long-pass filter (cut-off at $186 \mathrm{~cm}^{-1}, \mathrm{OD}>6 @ 532 \mathrm{~nm}$ ) was used to block the excitation light signal and to distinguish the scattered Raman signal. The signals were coupled into a fiber with a diameter of $100 \mu \mathrm{m}$ and analyzed using a high-resolution $\left(<8 \mathrm{~cm}^{-1}\right)$ spectrometer. To probe the Raman scattering of micro-modification, we measured the spectra along a $28-\mu \mathrm{m}$-line, which passed though the center of the ablation crater, covering all the modified area, with a spatial interval of $1 \mu \mathrm{m}$ controlled by the motorized stage. First, we have inspected the areas around the ablated crater via Raman scattering spectra and confirmed the absence of possible impurities induced by fs laser pulses (see supplementary material). Typical Raman spectra of graphene in the spectral range of $1200 \mathrm{~cm}^{-1}$ to $2900 \mathrm{~cm}^{-1}$ consist of D, G, and 2D bands, whose peaks usually are located at $\sim 1350 \mathrm{~cm}^{-1}$ (D band), $\sim 1580 \mathrm{~cm}^{-1}$ (G band), and $\sim 2680 \mathrm{~cm}^{-1}$ (2D band), respectively. Figure 2(a) shows a Raman spectrum of the CVD pristine SLG sample, in which the D, G, and 2D bands are marked. From the spatially resolved Raman measurements, a part of the spectra, corresponding to the whole $\mathrm{G}$ band from $1500 \mathrm{~cm}^{-1}$ to $1650 \mathrm{~cm}^{-1}$, were extracted and re-plotted as a contour map [Fig. 2(b)] according to their spatial positions. Figure 2(c) shows an enlarged view of the region marked by the dashed line in Fig. 2(b). In this figure, several areas with different spectral signatures (peak centers and widths) can be distinguished at different spatial positions. These regions are labeled regions I to IV and marked in Fig. 2(c) from the ablation crater to the outside. Figure 2(d) shows a sketch map of I-IV regions, which correspond to different spatial modification areas. The same spectral treatment was applied to the D band $\left(1250 \mathrm{~cm}^{-1}\right.$ to $\left.1450 \mathrm{~cm}^{-1}\right)$ and the $2 \mathrm{D}$ band (from $2550 \mathrm{~cm}^{-1}$ to $2800 \mathrm{~cm}^{-1}$ ), and the contour maps are shown in Figs. 3(a) and 3(b), respectively. Again, spectral changes can be observed in 2D and D bands for different spatial positions, including the peak height and width and spectral red- or blueshifting.

To further reveal the effect of the micro-modification, nonlinear curve fittings based on the Lorentz-peak function were utilized to obtain heights, widths, and center positions
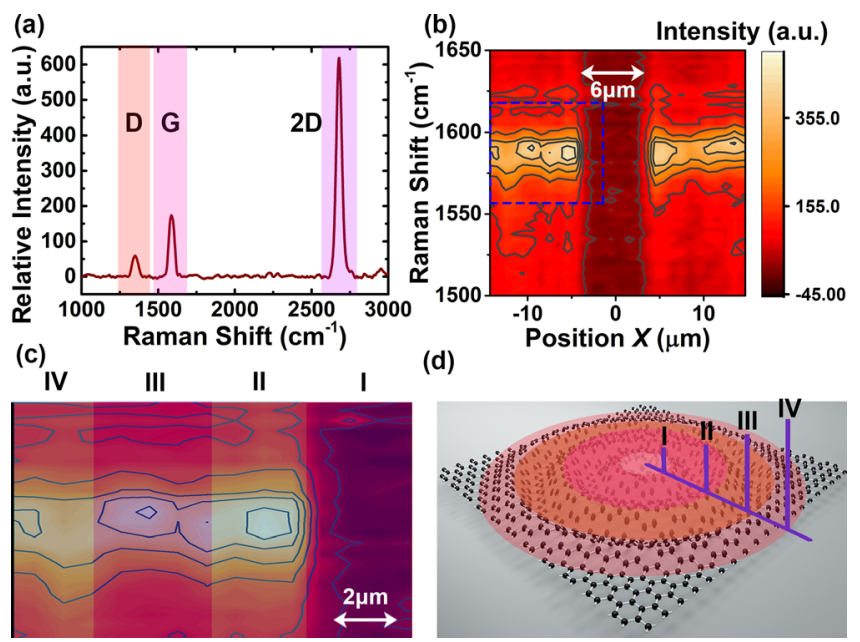

(d)

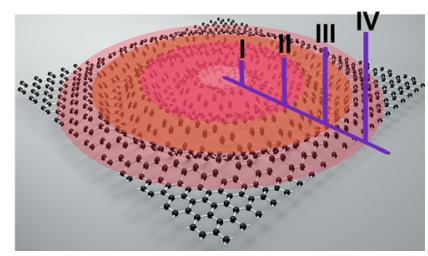

FIG. 2. A typical SLG Raman spectrum (a) without fs irradiation; a contour map cut from the $\mathrm{G}$ band spectrum (b) and its magnified area (c) at the fs irradiated spot; a schematic diagram (d) for regions I to IV. 
(a)

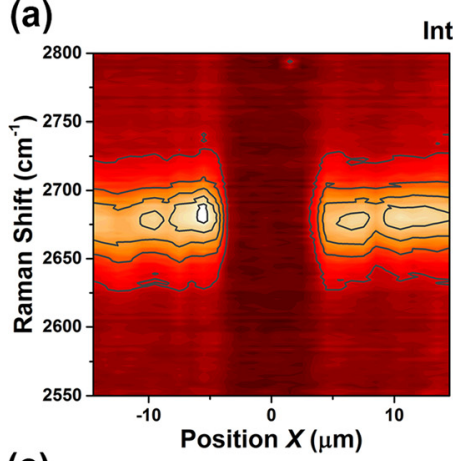

(c)

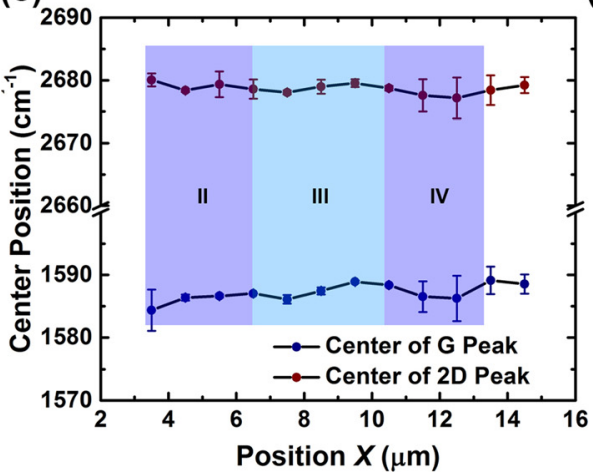

Intensity (a.u.) (b)

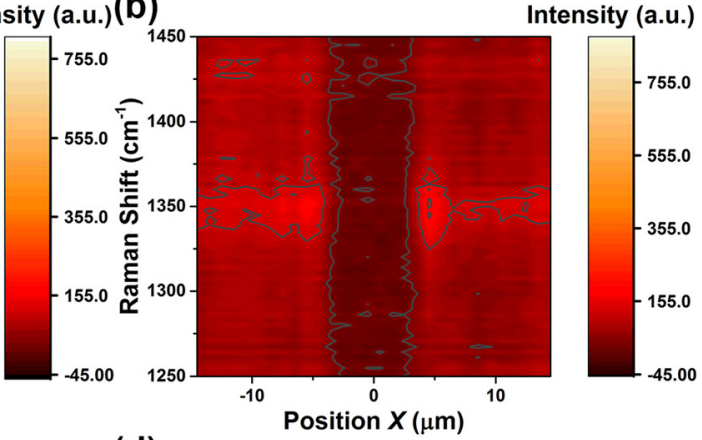

(d)

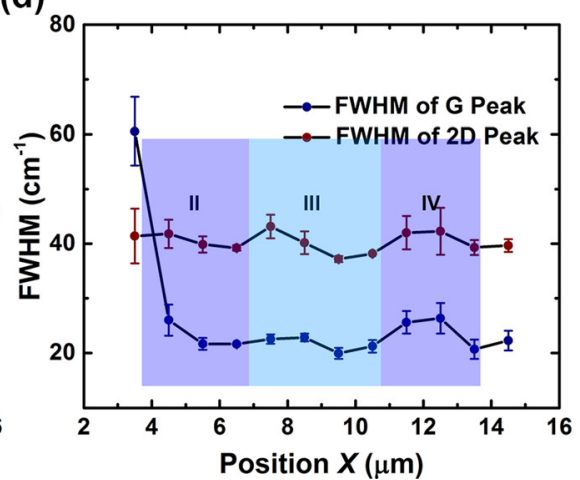

FIG. 3. Contour maps of 2D band (a) and D band (b) spectra; center positions (c) and full width at half maximum (FWHM) (d) of both bands. of the peaks of $\mathrm{G}, 2 \mathrm{D}$, and $\mathrm{D}$ bands, respectively. Figures 3 (a) and 3(b) show contour maps of 2D and D bands, respectively. The fitted center positions and full width at half maximum (FWHM) of $\mathrm{G}$ and 2D peaks at different positions are shown in Figs. 3(c) and 3(d), respectively. The segmented regions were labeled by block symbols, which were consistent with regions of Fig. 2(c). The ratios of fitted 2D (D) and $\mathrm{G}$ peak intensities [abbreviated as $I_{2 D} / I_{G}\left(I_{D} / I_{G}\right)$ ] were calculated and are shown in Fig. 4(a).

Based on the obtained spectra, the locations of regions I to IV were defined from the ablation crater to $0-\sim 3 \mu \mathrm{m}$ (I), $\sim 3 \mu \mathrm{m}$ to $6.5 \mu \mathrm{m}$ (II), $\sim 6.5 \mu \mathrm{m}$ to $10.5 \mu \mathrm{m}$ (III), and $\sim 10.5 \mu \mathrm{m}$ to $13.5 \mu \mathrm{m}$ (IV) along the radial direction. Compared to the pristine SLG sample, a series of spectral changes have been induced in region II, including FWHM of G peak [FWHM $(G)]$ broadening, the blue-shifting center position of $G$ peaks $[\operatorname{Pos}(\mathrm{G})]$, the red-shifting center position of the 2D peak [Pos(2D)], decreased $I_{2 D} / I_{G}$, and increased $I_{D} / I_{G}$. First, it was illustrated that the number of layers in region II may increase according to the Pos(2D) red-shifting [Fig. 3(c)] and the ratio of $I_{2 D} / I_{G}$ decreases rapidly [Fig. 4(a)]. ${ }^{33}$ Combined with the AFM image (an obvious bulge appears on the location of region II), it can be observed that the laser irradiation induced a folded graphene structure and a mass of defects. In this region, a 3- $\mu$ m-width ring area was covered by folded graphene, where the maximum ratio of $I_{2 D} / I_{G}$ is $\sim 1: 1$, which was close to the value of CVD bi-layer graphene (BLG). ${ }^{34}$ On the other hand, the ratios of $I_{D} / I_{G}$ varied inversely with the crystal size, which means that defects were induced. Thus, an average inter-defect distance $L_{D}$ and a defect density $n_{D}^{2}$ demonstrate the level of defects and disorder of graphene, which is expressed as ${ }^{32,35}$

$$
L_{D}^{2}=\frac{4.3 \times 10^{3}}{E_{L}^{4}}\left[\frac{I(D)}{I(G)}\right]^{-1},
$$

where $E_{L}$ is the excitation energy. In terms of defect density $n_{D}^{2}=1 /\left(\pi L_{D}^{2}\right)$,

$$
n_{D}^{2}=7.3 \times 10^{-9} E_{L}^{4} \frac{I(D)}{I(G)} .
$$

In region II, the values of $L_{D}$ and $n_{D}^{2}$ were calculated to be from 12.5 to $24.2 \mathrm{~nm}$ and from 53.8 to 199.5 [all the values are shown in Fig. 4(b)]. The defect distance was as short as $12.5 \mathrm{~nm}$ at the position close to the crater, therewith increasing rapidly with the position along the radial direction (a)

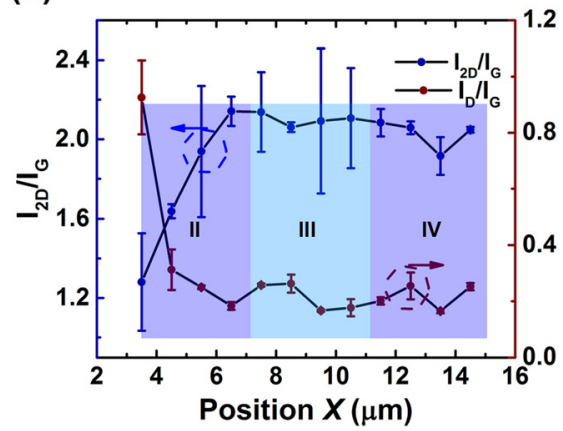

(b)

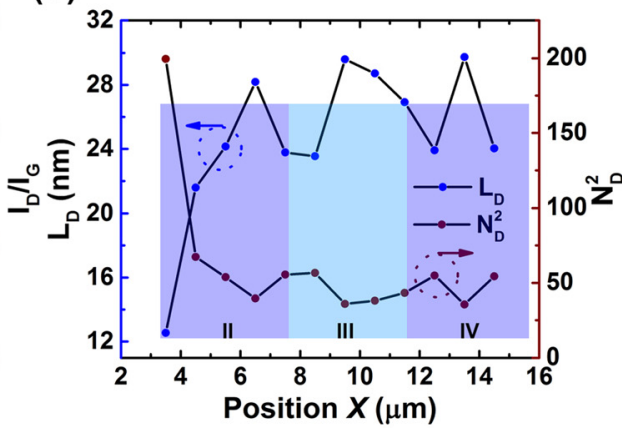

FIG. 4. Ratios of $I_{D} / I_{G}$ and $I_{2 D} / I_{G}$ (a) and calculated $L_{D}$ and $n_{D}^{2}$ (b) at different spatial positions. 
(a)

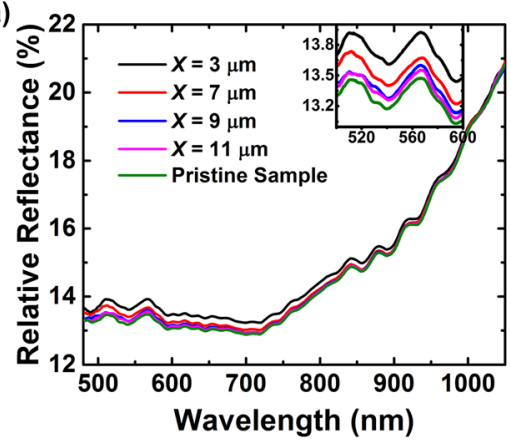

(b)

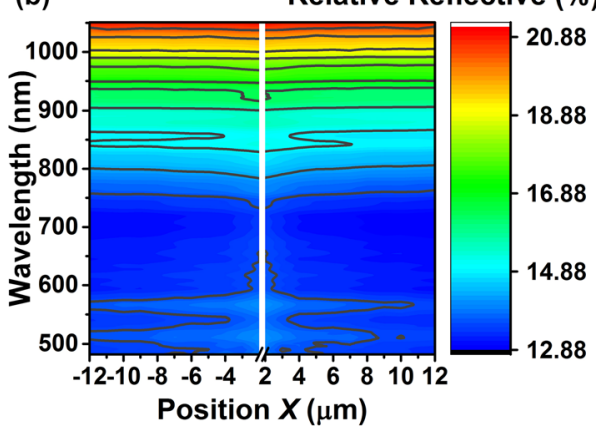

FIG. 5. Spectra of relative reflectance at positions $X=3,7,9$, and $11 \mu \mathrm{m}$ and pristine sample(a); the inset plot is a magnified spectral segment with wavelengths from 500 to $600 \mathrm{~nm}$. (b) A contour map corresponding to different positions from -12 to $12 \mu \mathrm{m}$. and decreasing gradually at the end of this region. The minimum defect distance has revealed that the SLG sample has turned to nano-crystalline graphene and still maintained the graphene lattice, which was larger than $3 \mathrm{~nm} .{ }^{36}$ Based on this effect, we can speculate that the folded graphene fragments have been gathered near the center and re-deposited to form a BLG. A mass of defects and disorders has been produced by this process, which also induced nano-crystallization in graphene.

For regions III and IV, we implemented the same analysis as for region II. In regions III and IV, instead of the folding effect found in region II, it has been observed that a large amount of debris has appeared on the surface, produced by the Coulomb explosion during the fs-laser ablation and maybe transported by the shock-wave. From the obtained spectra, it was reasonable to conclude that the re-deposition of graphene was negligible. According to undulation of the curve in Fig. 4(b), the fragmentations and defects have still been produced by such an adhesion process. The values of the defect distance and density have been calculated to be 23.5 to $29.6 \mathrm{~nm}$ and 35.8 to 56.6 (23.9 to $29.7 \mathrm{~nm}$ and 35.5 to 54.9) in region III (IV), respectively.

A homemade reflective micro-spectrometer was built with an optical microscope illuminated with a halogen lamp, and a spectrometer was placed at the conjugate plane of the sample. The incident light was projected on the sample surface, and the reflected light from the sample surface was collected into the spectrometer (measured range of 300 to $1100 \mathrm{~nm}$ ). Other experimental arrangements were the same as the micro-Raman setup except that the long-pass filter was removed. The collected spectral data were normalized considering the reflectivity of sapphire substrate as $100 \%$. Therefore, we obtained the reflective ratios of graphene and the sapphire substrate as a spectrum at the effective wavelength from $\sim 500$ to $1050 \mathrm{~nm}$. Figure 5(a) shows such spectra for the positions $X=3,7,9$, and $11 \mu \mathrm{m}$ and the pristine sample, with the inset showing the magnified segment from 500 to $600 \mathrm{~nm}$. Figure 5(b) presents a contour map with the reflective spectra arranged at different positions $X$ from -12 to $12 \mu \mathrm{m}$ (zero-position corresponding to the center of the ablation crater). A series of contour line changes have been observed in the map, which reveals that the relative reflectance of micro-modifications corresponding to the sapphire substrate was varying, indicating intrinsic correlation with the obtained micro-Raman spectra. In addition, the results also suggest that the refractive indices, which were relative to the substrate, have been modified in the investigated areas.
In summary, we have studied the optical effects of fs-laser irradiated graphene at the micrometric scale by micro-Raman spectroscopy. Permanent micro-modifications caused by fs pulses provided a measurable optical signature in the Raman spectrum of graphene. The presence of periodic structural modifications has been detected in surroundings of the ablation crater induced by fs pulses at the SLG. The induced structures consisted of four regions that were correlated with different mechanisms. These four regions have been characterized by micro-Raman scattering, which were coincident with data from the AFM, SEM, and Microreflectance investigations. This work may be helpful for the development of graphene-based microstructures or devices by fs laser pulses.

See supplementary material for the Raman scattering contour maps of the graphene on the sapphire sample after the fs-laser irradiation.

This work was supported by the National Natural Science Foundation of China (No. 11535008), Junta de Castilla y León (Project SA046U16), and MINECO (FIS2013-44174-P, FIS2015-71933-REDT).

${ }^{1}$ A. K. Geim and K. S. Novoselov, Nat. Mater. 6, 183 (2007).

${ }^{2}$ K. S. Novoselov, A. K. Geim, S. V. Morozov, D. Jiang, Y. Zhang, S. V. Dubonos, I. V. Grigorieva, and A. A. Firsov, Science 306, 666 (2004).

${ }^{3}$ A. K. Geim, Science 324, 1530 (2009).

${ }^{4}$ A. A. Balandin, S. Ghosh, W. Bao, I. Calizo, D. Teweldebrhan, F. Miao, and C. N. Lau, Nano Lett. 8, 902 (2008).

${ }^{5} \mathrm{Z}$. Li and F. Chen, Appl. Phys. Rev. 4, 011103 (2017).

${ }^{6}$ J.-H. Yoo, E. Kim, and D. J. Hwang, MRS Bull. 41, 1002 (2016).

${ }^{7}$ A. Gil-Villalba, C. Xie, R. Salut, L. Furfaro, R. Giust, M. Jacquot, P. A. Lacourt, J. M. Dudley, and F. Courvoisier, Appl. Phys. Lett. 107, 061103 (2015).

${ }^{8}$ L. Guo, Y.-L. Zhang, D.-D. Han, H.-B. Jiang, D. Wang, X.-B. Li, H. Xia, J. Feng, Q.-D. Chen, and H.-B. Sun, Adv. Opt. Mater. 2, 120 (2014).

${ }^{9}$ I. Paradisanos, E. Kymakis, C. Fotakis, G. Kioseoglou, and E. Stratakis, Appl. Phys. Lett. 105, 041108 (2014).

${ }^{10}$ W. Nie, R. He, C. Cheng, U. Rocha, J. R. Vazquez de Aldana, D. Jaque, and F. Chen, Opt. Lett. 41, 2169 (2016).

${ }^{11}$ T. Calmano, A.-G. Paschke, J. Siebenmorgen, S. T. Fredrich-Thornton, H. Yagi, K. Petermann, and G. Huber, Appl. Phys. B 103, 1 (2011).

${ }^{12}$ J. Lamela, D. Jaque, A. Rodenas, F. Jaque, G. A. Torchia, J. R. Vazquez, C. Mendez, and L. Roso, Appl. Phys. A 93, 177 (2008).

${ }^{13}$ R. R. Gattass and E. Mazur, Nat. Photonics 2, 219 (2008).

${ }^{14}$ Y. Zhang, G. Li, S. Wei, Y. He, H. Xia, Q. Chen, H.-B. Sun, and F.-S. Xiao, Nano Today 5, 435 (2010).

${ }^{15}$ D. Tan, S. Zhou, B. Xu, P. Chen, Y. Shimotsuma, K. Miura, and J. Qiu, Carbon 62, 374 (2013).

${ }^{16}$ J. B. Park, W. Xiong, Y. Gao, M. Qian, Z. Q. Xie, M. Mitchell, Y. S. Zhou, G. H. Han, L. Jiang, and Y. F. Lu, Appl. Phys. Lett. 98, 123109 (2011).

${ }^{17}$ M. Lenner, A. Kaplan, and R. E. Palmer, Appl. Phys. Lett. 90, 153119 (2007). 
${ }^{18}$ C. Li, X. Kang, Q. Zhu, and W. Zheng, Appl. Surf. Sci. 398, 89 (2017).

${ }^{19}$ D. Tan, X. Liu, Y. Dai, G. Ma, M. Meunier, and J. Qiu, Adv. Opt. Mater. 3, 836 (2015).

${ }^{20}$ R. Sahin, E. Simsek, and S. Akturk, Appl. Phys. Lett. 104, 053118 (2014).

${ }^{21}$ A. Roberts, D. Cormode, C. Reynolds, T. Newhouse-Illige, B. J. LeRoy, and A. S. Sandhu, Appl. Phys. Lett. 99, 051912 (2011).

${ }^{22}$ R. Trusovas, K. Ratautas, G. Račiukaitis, J. Barkauskas, I. Stankevičienė, G. Niaura, and R. Mažeikienè, Carbon 52, 574 (2013).

${ }^{23}$ J.-H. Yoo, J. Bin In, J. Bok Park, H. Jeon, and C. P. Grigoropoulos, Appl. Phys. Lett. 100, 233124 (2012).

${ }^{24}$ M. Currie, J. D. Caldwell, F. J. Bezares, J. Robinson, T. Anderson, H. Chun, and M. Tadjer, Appl. Phys. Lett. 99, 211909 (2011).

${ }^{25}$ R. J. Stöhr, R. Kolesov, K. Xia, and J. Wrachtrup, ACS Nano 5, 5141 (2011).

${ }^{26}$ W. Xiong, Y. S. Zhou, W. J. Hou, L. J. Jiang, Y. Gao, L. S. Fan, L. Jiang, J. F. Silvain, and Y. F. Lu, Sci. Rep. 4, 4892 (2014).
${ }^{27}$ X. C. Wang, G. C. Lim, H. Y. Zheng, F. L. Ng, W. Liu, and S. J. Chua, Appl. Surf. Sci. 228, 221 (2004).

${ }^{28}$ A. H. Nejadmalayeri and P. R. Herman, Opt. Express 15, 10842 (2007).

${ }^{29}$ S. Kanehira, J. Si, J. Qiu, K. Fujita, and K. Hirao, Nano Lett. 5, 1591 (2005).

${ }^{30}$ M. R. Tejerina, D. Jaque, and G. A. Torchia, J. Appl. Phys. 112, 123108 (2012).

${ }^{31}$ A. C. Ferrari, Solid State Commun. 143, 47 (2007).

${ }^{32}$ A. C. Ferrari and D. M. Basko, Nat. Nanotechnol. 8, 235 (2013).

${ }^{33}$ A. C. Ferrari, J. C. Meyer, V. Scardaci, C. Casiraghi, M. Lazzeri, F. Mauri, S. Piscanec, D. Jiang, K. S. Novoselov, S. Roth, and A. K. Geim, Phys. Rev. Lett. 97, 187401 (2006).

${ }^{34}$ A. Reina, X. Jia, J. Ho, D. Nezich, H. Son, V. Bulovic, M. S. Dresselhaus, and J. Kong, Nano Lett. 9, 30 (2009).

${ }^{35}$ L. G. Cançado, A. Jorio, E. H. Martins Ferreira, F. Stavale, C. A. Achete, R. B. Capaz, M. V. O. Moutinho, A. Lombardo, T. S. Kulmala, and A. C. Ferrari, Nano Lett. 11, 3190 (2011).

${ }^{36}$ A. C. Ferrari and J. Robertson, Phys. Rev. B 61, 14095 (2000). 